MATHEMATICS OF COMPUTATION

Volume 73, Number 245, Pages 415-423

S 0025-5718(03)01537-0

Article electronically published on May 7, 2003

\title{
REAL ZEROS OF REAL ODD DIRICHLET $L$-FUNCTIONS
}

\author{
MARK WATKINS
}

\begin{abstract}
Let $\chi$ be a real odd Dirichlet character of modulus $d$, and let $L(s, \chi)$ be the associated Dirichlet $L$-function. As a consequence of the work of Low and Purdy, it is known that if $d \leq 800000$ and $d \neq 115147,357819$, 636184 , then $L(s, \chi)$ has no positive real zeros. By a simple extension of their ideas and the advantage of thirty years of advances in computational power, we are able to prove that if $d \leq 300000000$, then $L(s, \chi)$ has no positive real zeros.
\end{abstract}

\section{INTRODUCTION}

Let $\chi$ be a primitive real Dirichlet character. We wish to determine whether the associated $L$-function $L(s, \chi)=\sum_{n=1}^{\infty} \frac{\chi(n)}{n^{s}}$ for $\Re s>0$ has any positive real zeros. By classical arguments, we have $L(s, \chi) \neq 0$ for $s \geq 1$, and by the functional equation, we need only consider $1 / 2 \leq s<1$. We shall restrict ourselves to odd characters, i.e. those such that $\chi(-1)=-1$, for reasons which will become clear. We remark that the best results for even $\chi$ have been obtained by Rosser, who, in unpublished work, showed that for moduli less than $986, L(s, \chi)$ has no positive real zeros. The sharpest theoretical results have been obtained by Conrey and Soundararajan [2], who show that asymptotically at least $20 \%$ of the primitive real odd Dirichlet characters $\chi$ with 8 dividing the modulus have $L(s, \chi)>0$ for $s>0$.

By well-known arguments, the assumptions that $\chi$ is odd, primitive, and has modulus $d>4$ implies that $\zeta(s) L(s, \chi)$ is the Dedekind zeta-function of the imaginary quadratic field of discriminant $-d$. In particular we have that $\chi(n)=(-d \mid n)$, where $(-d \mid n)$ is the Kronecker symbol. For $\Re s>1$, we also have

$$
\zeta(s) L(s, \chi)=\sum_{(a, b, c) \in Q} Z(s ; a, b, c),
$$

where

$$
Q=\left\{(a, b, c) \in \mathbf{Z}^{3} \mid-d=b^{2}-4 a c,-a<b \leq a<c \text { or } 0 \leq b \leq a=c\right\}
$$

is the set of reduced integral solutions of $-d=b^{2}-4 a c$, and

$$
Z(s ; a, b, c)=\frac{1}{2} \sum_{(m, n) \neq(0,0)} \sum_{\left(a m^{2}+b m n+c n^{2}\right)^{s}} .
$$

Low [3] found three conditions which, if satisfied for a given modulus $d$, imply that $L(s, \chi)$ has no positive real zeros. Using an IBM 7094, he verified these conditions for moduli less than 593000 , finding one exception to his first condition. In his

Received by the editor February 14, 2002 and, in revised form, May 29, 2002.

2000 Mathematics Subject Classification. Primary 11M20; Secondary 11M06.

(C)2003 American Mathematical Society 
review of Low's work, Rieger [5] stated that an error analysis was lacking. To remedy the problem, Purdy [4] extended Low's work to moduli less than 800000 and performed an accompanying error analysis. Purdy found three exceptions to Low's first condition: 115147,357819 , and 636 814. By a slight modification of Low's argument, we can overcome the fact that these three moduli fail his first condition. This is somewhat important, as many references to Low's work sloppily ignore this point. Moreover, due to thirty years of advances in computational power, we can extend these results up to moduli less than 300000000 . We shall show the following:

Theorem. Let $\chi$ be a real odd Dirichlet character of modulus $d \leq 300000000$. Then $L(s, \chi)$ has no positive real zeros.

\section{LOW'S THREE CONDITIONS}

We let $-d$ be a fundamental discriminant with $d>4$, and let $Q$ be the aforementioned set of reduced integral solutions of $-d=b^{2}-4 a c$. We define

$$
\alpha(a, d)=\log a+\log \left(8 \pi e^{-\gamma}\right)-\frac{1}{2} \log d,
$$

where $\gamma$ is Euler's constant. We define $b_{n}$ and $\beta_{n}$ from the relations (for $|s-1|<3$ )

$$
\log [(s-1) \zeta(s)]=\sum_{n=1}^{\infty}(-1)^{n} \beta_{n}(s-1)^{n} \quad \text { and } \quad b_{n}=\left(2^{n}-1\right) \frac{\zeta(n)}{n}+2^{n} \beta_{n} .
$$

We list some properties of the $b_{n}$ :

$$
\begin{gathered}
b_{n}<2^{n+1}, \\
2.09<b_{2}<2.10, \quad 2.66<b_{3}<2.67, \quad 3.99<b_{4}<4.0, \quad 6.39<b_{5}<6.4, \\
b_{6}<10.67, \quad 18.28<b_{7}<18.29, \quad 56.88<b_{9}<56.89, \quad b_{11}<186.19, \\
b_{2 n+1}>594 \quad \text { for } n \geq 6 .
\end{gathered}
$$

A proof of (2) appears in Low's work, (3) can be verified directly, and (4) follows from Low's statement that $b_{n}>\frac{2^{n}}{n}\left(1-7.44(2 / 3)^{n-1}\right)$ for $n \geq 6$.

From [1, it is known that the analytic continuation of $Z(s ; a, b, c)$ satisfies

$$
\frac{1}{\sqrt{k}}\left(\frac{a k}{\pi}\right)^{s} \Gamma(s) Z(s ; a, b, c)=f(s)+f(1-s)+H(s ; a, b, c),
$$

where $k=\sqrt{d} / 2 a$ and $f(s)=(k / \pi)^{s} \Gamma(s) \zeta(2 s) / \sqrt{k}$ and $H(s ; a, b, c)$ is an error term given by

$$
H(s ; a, b, c)=4 \sum_{n=1}^{\infty} n^{s-1 / 2} \sigma_{1-2 s}(n) \cos \left(\frac{\pi n b}{a}\right) K_{s-1 / 2}(2 \pi k n),
$$

where $\sigma_{l}(n)=\sum_{m \mid n} m^{l}$ and $K_{l}(z)$ is the standard $K$-Bessel function given by $K_{l}(z)=\int_{0}^{\infty} e^{-z \cosh t} \cosh l t d t$. 
The three conditions of Low are

$$
\begin{aligned}
& \text { (i) } S_{0}=\sum_{Q} \frac{\alpha(a, d)}{\sqrt{a}} \geq 0, \\
& \text { (ii) } U(s)=\sum_{Q} \frac{\alpha(a, d)-H(s ; a, b, c)}{\sqrt{a}}>0 \quad \text { for } 1 / 2 \leq s<1, \\
& \text { (iii) } S_{n}=\sum_{Q} \frac{1}{\sqrt{a}}\left(b_{2 n+1}+\frac{\alpha^{2 n+1}(a, d)}{(2 n+1) !}\right) \geq 0 \quad \text { for } n \geq 1 .
\end{aligned}
$$

It is proven in 3 that these imply $L(s, \chi)>0$ for $0<s<1$. Note that (4) implies that (iii) trivially holds for sufficiently large $n$. In fact, the following lemma allows us to check condition (iii) quite efficiently.

Lemma. Assume that $d \leq 10^{10}$. Then $S_{n} \geq 0$ for $n \geq 6$, and $S_{1} \geq 0$ and $S_{2} \geq 0$ imply that $S_{n} \geq 0$ for all $n \geq 1$.

Since $1 \leq a \leq \sqrt{d}$, our assumption that $d \leq 10^{10}$ combined with (1) gives us that $-\sqrt{80} \leq \alpha(a, d) \leq 3$ for all $(a, d)$ pairs. So for $n \geq 6$, we can use (4) to conclude that

$$
b_{2 n+1}+\frac{\alpha^{2 n+1}(a, d)}{(2 n+1) !} \geq 594-\frac{(\sqrt{80})^{13}}{13 !} \geq 200 .
$$

Thus $S_{n} \geq 0$ for $n \geq 6$, since every term in the sum is positive. For the $n=3$, $n=4$, and $n=5$ terms we can exploit the behavior of certain polynomials. We have that

$$
\frac{x^{7}}{7 !}-\frac{8}{3} \frac{x^{5}}{5 !}+b_{7}-\frac{8}{3} b_{5} \geq 0
$$

for $-\sqrt{80} \leq x \leq 3$. This follows from the fact that the critical points of the polynomial are at $x=0$ and $x= \pm \sqrt{80}$. Evaluating at the critical points and using (3) gives the desired inequality. Letting $x$ be $\alpha(a, d)$, multiplying by $1 / \sqrt{a}$ and summing over $Q$, this implies that $S_{3} \geq \frac{8}{3} S_{2} \geq 0$. Similarly,

$$
\frac{x^{9}}{9 !}-\frac{3}{2} \frac{x^{7}}{7 !}+b_{9}-\frac{3}{2} b_{7} \geq 0
$$

for $-\sqrt{80} \leq x \leq 3$, and so $S_{4} \geq \frac{3}{2} S_{3} \geq 0$. Finally,

$$
\frac{x^{11}}{11 !}-\frac{x^{9}}{9 !}+b_{11}-b_{9} \geq 0
$$

for $-\sqrt{80} \leq x \leq 3$, and so $S_{5} \geq S_{4} \geq 0$. Thus if $S_{1} \geq 0$ and $S_{2} \geq 0$ and $d \leq 10^{10}$, we see that (iii) holds for all $n$, proving the Lemma.

In our range of moduli, the failures of Low's method come mostly from (i). A failure of (ii) would be very good evidence against GRH, while failures of (iii) might occur for the same spurious reasons that (i) fails for certain moduli. For moduli $d \leq 300000000$, we found that (ii) is always true, while (i) fails for 4507 moduli. One failure of (iii) was found: $-0.25<S_{2}<-0.23$ for $d=290661067$. In the sequel, we shall have to deal with this modulus separately. 


\section{Dealing with failures to Condition (i) In the RAnge $d \leq 10^{10}$}

We follow the proof of [2, Theorem 2]. For $|r|<1 / 2$, we have

$$
\sum_{Q} \frac{1}{\sqrt{a}}[\alpha(a, d)+m(r)] h(r ; a, d) \geq S_{0}+\sum_{n=1}^{\infty} S_{n} r^{2 n}
$$

where

$$
m(r)=\sum_{n=1}^{\infty} b_{2 n+1} r^{2 n}, \quad g(r)=r[\alpha(a, d)+m(r)]
$$

and

$$
h(r ; a, d)=\frac{\sinh [g(r)]}{g(r)}=\sum_{n=0}^{\infty} \frac{g^{2 n}(r)}{(2 n+1) !} .
$$

We now show how to deal with failures to condition (i), and thus we shall assume that $S_{0}<0$. We shall also assume that (ii) and (iii) hold. We want to multiply the left side of (5) by $l(r)=\exp \left(\sum_{n=1}^{\infty} b_{2 n} r^{2 n}\right) \geq 1$, and if $S_{0}+S_{1} r^{2} \geq 0$, this will yield the valid inequality

$$
\sum_{Q} \frac{l(r)}{\sqrt{a}}[\alpha(a, d)+m(r)] h(r ; a, d) \geq S_{1} r^{2}+S_{0} \geq S_{0} .
$$

This is in contrast to Low, who always required $S_{0} \geq 0$, which precluded him from being able to handle the cases when $S_{0}<0$. So for

$$
|r| \geq \sqrt{-S_{0} / S_{1}}
$$

we see that (6) holds. As in Low's proof, (6) and (ii) imply that $L(s, \chi)>0$ for $s=0.5+r$ for $r$ in the range (7). So if $S_{0}<0$, we can conclude that $L(s, \chi) \neq 0$ for $s>0.5+\sqrt{-S_{0} / S_{1}}$. For our range of $d$, our computations show that (when $\left.S_{0}<0\right)$ we always have $\sqrt{-S_{0} / S_{1}}<0.05$, and so we need only consider $|r| \leq 0.05$ in the remainder of this section. Because of numerical round-off errors which may occur, we shall actually consider all $S_{0} \leq 0.02$ instead of just negative $S_{0}$. This will not be a big issue; our main goal will be to show (6) for certain ranges of $r$.

We see that (6) holds trivially at $r=0$, and we hope that by taking a power series expansion around $r=0$, we will find that (6) holds in a region which overlaps (7). Since the $b_{i}$ are nonnegative, to show (6), it suffices to show that

$$
\sum_{Q} \frac{l(r)}{\sqrt{a}}\left[\alpha(a, d)+b_{3} r^{2}+b_{5} r^{4}\right] h(r ; a, d) \geq S_{0} .
$$

Our computations show that $\left|S_{0}\right| \leq 1 / 50$ and $S_{1} \geq 1$ for all moduli $d$ in our range for which $S_{0} \leq 1 / 50$, and so we will assume these inequalities for the remainder of the section. Using $0 \leq b_{n} \leq 2^{n+1},|r| \leq 1 / 20$, and (3), we get that

$$
\sum_{n \geq 3} b_{2 n} r^{2 n} \leq 12 r^{6}
$$

and

$$
\begin{aligned}
1+b_{2} r^{2}+\left(b_{4}+b_{2}^{2} / 2\right) r^{4} & \leq l(r) \leq \exp \left(b_{2} r^{2}+b_{4} r^{4}+12 r^{6}\right) \\
& \leq 1+b_{2} r^{2}+\left(b_{4}+b_{2}^{2} / 2\right) r^{4}+23 r^{6}
\end{aligned}
$$


which gives $l(r)=1+b_{2} r^{2}+\left(b_{4}+b_{2}^{2} / 2\right) r^{4}+23 \theta r^{6}$ for some $|\theta| \leq 1$. We make the abbreviation $\alpha=\alpha(a, d)$ and note that

$$
-\sqrt{80} \leq \alpha \leq \frac{g(r)}{r}=\alpha+\sum_{n=1}^{\infty} b_{2 n+1} r^{2 n} \leq \alpha+\frac{4}{99} \leq 3+\frac{4}{99}
$$

which gives that $g^{2}(r) \leq 80 r^{2} \leq 1 / 5$, which implies

$$
\sum_{n=3}^{\infty} \frac{g^{2 n}(r)}{(2 n+1) !} \leq 80^{3} r^{6} \sum_{n=3}^{\infty} \frac{1}{5^{n-3}(2 n+1) !} \leq 102 r^{6} .
$$

By a similar bounding of tails, we can obtain the results that

$$
g^{2}(r)=\left(\alpha r+b_{3} r^{3}+7 \theta r^{5}\right)^{2} \quad \text { and } \quad g^{4}(r)=\left(\alpha r+3 \theta r^{3}\right)^{4}
$$

for some $|\theta| \leq 1$, which need not be the same at every appearance. Thus we obtain that

$$
h(r ; a, d)=1+\sum_{n=1}^{\infty} \frac{g^{2 n}(r)}{(2 n+1) !}=1+\frac{\alpha^{2}}{6} r^{2}+\left(\frac{b_{3} \alpha}{3}+\frac{\alpha^{4}}{120}\right) r^{4}+200 \theta r^{6} .
$$

In the last step we have used $|\alpha| \leq 9$. Expanding out (8), we get that the left side is

$$
\begin{aligned}
\sum_{Q} \frac{1}{\sqrt{a}}\left(1+b_{2} r^{2}+\right. & \left.\left(b_{4}+b_{2}^{2} / 2\right) r^{4}+23 \theta r^{6}\right)\left[\alpha(a, d)+b_{3} r^{2}+b_{5} r^{4}\right] \\
& \times\left(1+\frac{\alpha^{2}(a, d)}{6} r^{2}+\left(\frac{b_{3} \alpha(a, d)}{3}+\frac{\alpha^{4}(a, d)}{120}\right) r^{4}+200 \theta r^{6}\right) .
\end{aligned}
$$

We wish to find a range for $r$ such that (9) is greater than $S_{0}$. We multiply out (9) in order to find a lower bound for it. There will be some obviously positive terms like $b_{3} \alpha^{2} r^{4} / 2 \sqrt{a}$ and $b_{2} b_{5} \alpha^{2} r^{8} / 6 \sqrt{a}$ which we can ignore. Our multiplication will also yield multiples of $S_{1}$ and $S_{2}$, such as $S_{1}\left(b_{4}+b_{2}^{2} / 2\right) r^{6}$ and $S_{2} b_{2} r^{6}$. Since these are positive, we can ignore them also. We define $T=\sum_{Q} 1 / \sqrt{a}$, and thus we obtain a lower bound of

$$
S_{0}+\left(S_{1}+b_{2} S_{0}\right) r^{2}+\left(S_{2}+b_{2} S_{1}+\left[b_{4}+b_{2}^{2} / 2\right] S_{0}\right) r^{4}-2100 T r^{6}
$$

for (9) by multiplying it out and using the bounds on the $b_{i}$ and $|\alpha|$. Since $\left|S_{0}\right| \leq$ $1 / 50$ and $S_{1} \geq 1$ for all of our failures of (i), the $r^{4}$ term in the above is positive, and so we can ignore it. Thus we find that we have (8) for

$$
|r| \leq \min \left(0.05,\left(\frac{S_{1}+b_{2} S_{0}}{2100 T}\right)^{1 / 4}\right) .
$$

If this overlaps the previous range (7) for $r$, we can conclude that $L(s, \chi)$ has no positive real zeros. Our program found that these ranges do overlap for all failures of (i) for $d \leq 300000000$. As an example, the smallest $S_{0}$ occurs for $d=223191759$, for which $S_{0} \approx-0.01638$. We have $S_{1} \geq 314$ and $T \leq 249$. Hence (7) implies (6) for $r \geq .0073$, while the range in (10) gives us (8) when $|r| \leq \min (.05, .156)$. 


\section{The modulus 290661067}

Even though condition (iii) fails for $n=2$ for this modulus, we can still use the ideas of the previous section to show that there are no positive real zeros of the associated $L$-function. Computations show that $S_{0} \geq 0, S_{1} \geq 3, S_{2} \geq-1 / 4$, $S_{3} \geq 250$, and $S_{4} \geq 1200$, while the argument of Section 3 shows that $S_{n} \geq 0$ for $n \geq 5$. By (5) we have

$$
\sum_{Q} \frac{1}{\sqrt{a}}[\alpha(a, d)+m(r)] h(r ; a, d) \geq S_{0}+S_{1} r^{2}+S_{2} r^{4} .
$$

In order to be able to obtain (6), we need both $S_{0}+S_{1} r^{2}+S_{2} r^{4} \geq 0$ and $S_{1} r^{2}+$ $S_{2} r^{4} \geq 0$. But for $|r|<1 / 2$, both of these are true due to the inequalities $S_{0} \geq 0$, $S_{1} \geq 3$, and $S_{2} \geq-1 / 4$. Thus we have (6), and so we only need to verify (ii) to conclude that $L(s, \chi)>0$ for real $s>0$ for the quadratic character modulo 290661067 . Such a verification was done as for the other moduli, as described in Section 5. We record the facts that this modulus is prime, and the class number of the associated imaginary quadratic field is 1101 . Also, the lowest height zero of the associated $L$-function appears to be at approximately $s=1 / 2+0.0477924709188 i$.

\section{How THE PROGRAMS WORKED}

The first thing to do is to generate members of $Q$, i.e. find solutions to $-d=$ $b^{2}-4 a c$ with certain bounds on the size of $a, b$, and $c$. The most expedient way to do this is to let $b$ run from 0 to $\sqrt{d / 3}$ and then factor $\left(b^{2}+d\right) / 4$ to find possible $(a, c)$ pairs. Of course, factoring can be a time-consuming process. To speed up the factoring step, we create an enormous array. In the $q$ th position of the array, we store the smallest prime factor of $q$, storing 0 if $q$ is prime. Such an array can be created very quickly by the use of sieve methods. The array needs to contain numbers up to $d / 3$. Of course, we need not consider even numbers in the array, since it is so easy to divide by two. Since the smallest prime divisor of a composite number is less than its square root and $d / 3$ is no more than $10^{8}$ for our range of moduli, we never have to store anything in our array greater than 10000, thus allowing the word size to be 16 bits. This allowed us to keep the memory requirements down, a great concern when dealing with an array with around $10^{8}$ elements.

Having created this array which allows fast factorization, the program next goes through the moduli $d$ in the range it is given. It checks that $d$ is in one of the six congruence classes $3,4,7,8,11$, or 15 modulo 16 , and also that 9,25 , and 49 do not divide $d$. We just ignore the fact that higher squares may divide $d$, as it would be too time-consuming to check this. For each $d$, we let $b$ run from 0 to $\sqrt{d / 3}$, and we find the factors of $\left(b^{2}+d\right) / 4$ by iteratively using our array to find the smallest prime factors. Note that unless $b=a$ or $a=c$ or $b=0$, a triple $(a, b, c)$ in $Q$ has a distinct "conjugate" in $Q$, namely $(a,-b, c)$. When we speak of doing operations on members of $Q$ in the sequel, it is understood that we only consider $b \geq 0$ and multiply by the necessary multiplicity. For $(a, b, c)$ triples in $Q$, we make lower bound estimates for the contributions to $S_{0}, S_{1}, S_{2}$, and $U(s)$. We describe these more fully in the next paragraphs.

Since the $\mathbf{C}$ library functions $\mathbf{l o g}$, exp, sqrt, and cos are rather time-consuming to compute, and for the vast majority of $d$ the conditions of Low will hold quite comfortably, it was decided that, as a first measure, crudely bounding these functions would be more efficacious in the long run, with sharper bounds then used 
only for the $d$ which were cantankerous. The only quantity of which we need to take the square root or natural logarithm many times is $a$, and we know that $a \leq \sqrt{d / 3} \leq 10000$. Thus three arrays are created at the beginning of the program, containing upper and lower bounds for $\sqrt{a}$ and lower bounds for $\log (a)$ for all integers $1 \leq a \leq 10000$. To check condition (ii), we also need to be able to upper bound $H(s ; a, b, c)$ easily, and by the results of [1] and [2, Theorem 4] we have

$$
H(s ; a, b, c) \leq \frac{2 \cos (\pi b / a)}{k^{1 / 2} e^{2 \pi k}}+\frac{1}{25} \frac{|2 \cos (\pi b / a)|}{k^{1 / 2} e^{2 \pi k}},
$$

where $k=\sqrt{d} / 2 a$. To expedite calculation of the terms involving $k$, two arrays are created which contain upper and lower bounds for the values of $k^{-1 / 2} e^{-2 \pi k}$ for $0 \leq k \leq 2$ at $k$-intervals of $1 / 25$. The upper bounds contain a factor of $2+\frac{1}{25}=2.08$, while the lower bounds carry a factor of 1.92 , as these quantities would appear in the calculations in any case. The program then simply rounds $k$ either up or down to the nearest demarcation and looks up the appropriate bound in the tables. The cosine is bounded by fourth order Taylor polynomials. If $2 b<a$, then upper bounds for $\cos (\pi b / a)$ and the expression involving $k$ are used, while if $2 b>a$, the corresponding lower bounds are taken.

For a given $d$, each member of $Q$ has its contribution to the four sums in question bounded in this manner. In order to calculate $\alpha(a, d)$, the value of $\log (d)$ is computed once with the $\mathbf{C}$ library function. Similarly, $\sqrt{d}$ is computed once with the $\mathbf{C}$ library function so that $k=\sqrt{d} / 2 a$ can be determined. The computations for a given $d$ can be ended early if all four sums are positive and it is ascertained that additional values of $b$ can only add nonnegative values to the four sums. The values of $b_{3}$ and $b_{5}$ that occur in $S_{1}$ and $S_{2}$ were pre-computed, and lower bounds for these are used in the program. Similarly, the value of $\log \left(8 \pi e^{-\gamma}\right)$ was computed once and a lower bound is used in the program.

Note that the repetitive computations of the program involved with individual members of $Q$ use nothing but the four basic operations, while the values in tables at the beginning can easily be checked against more accurate computations to bound their error. It is therefore a straightforward but tedious task to undertake an error analysis as in [4. In practice, any $d$ with computed bounds for $S_{0}, S_{1}, S_{2}$ or $U(s)$ which are less than 0.001 are written to an output file, which is sufficient to compensate for any accumulated round-off error. As mentioned above, for all moduli we considered we had $S_{1} \geq 0$, and $S_{2}$ was positive for all $d$ save 290661067 . Note that any modulus $d$ which is not written to the output file satisfies all of Low's conditions, and thus we can conclude that the corresponding $L$-function has no positive real zeros.

The moduli which remain are then handled by a more robust program, using a multi-precision package. To put the level of precision in perspective, we note that the smallest lower bound for $U(s)$ in our range of $d$ is $2.073 \times 10^{-6}$ for the modulus $d=175990483$. This secondary program computes the sum $T=\sum_{Q} 1 / \sqrt{a}$, which appeared in Section 3, and also checks to see if $d$ has a nontrivial odd square factor (recall that previously we had only checked this for 9, 25, and 49; for instance, $d=13932787$ will fail the first program, but it is divisible by 121). There were 4507 moduli with $S_{0}<0$ which got passed to this secondary program, of which 64 were eliminated by having a nontrivial odd square factor. For each of the 4443 remaining moduli, it is relatively easy to verify that the ranges (7) and (10) of Section 3 overlap, but condition (ii) can often be difficult to verify, since the $L$-function is so 
small near its central point in many of these cases. As a first measure, we do not make a time-consuming effort to get an impressive upper bound on $H(s ; a, b, c)$, but we are less crude than previously. In fact, often the crude bound of the first program is enough to conclude that $U(s)$ is positive (throughout the desired range), but the secondary program still verified this anyway. If our lower bound for $U(s)$ is still negative, then a heavy-duty tertiary program with even sharper bounds and more precision is used (these was necessary for a total of nine moduli).

We describe the estimates used in the generic secondary program. We write $v=s-1 / 2$, and from $[3$, we have that

(11) $H(s ; a, b, c) \leq 4 \cos \left(\frac{\pi b}{a}\right) K_{v}(2 \pi k)+4\left[2^{v}+2^{-v}\right] \cos \left(\frac{2 \pi b}{a}\right) K_{v}(4 \pi k)+\frac{7}{\sqrt{k} e^{6 \pi k}}$.

We need only consider $v$ in the range $0 \leq v<1 / 2$, and in this range from [1] we have (for all positive integers $n$ )

$$
1-\frac{1-4 v^{2}}{16 \pi n k} \leq 2(k n)^{1 / 2} e^{2 \pi k n} K_{v}(2 \pi k n) \leq 1 .
$$

Thus if $\cos (\pi b / a)$ is positive, then the first term in (11) is no greater than $2 \cos (\pi b / a) / \sqrt{k} e^{2 \pi k}$, while if $\cos (\pi b / a)$ is negative, the corresponding bound is

$$
\frac{2 \cos (\pi b / a)}{\sqrt{k} e^{2 \pi k}}\left[1-\frac{1}{16 \pi k}\right] \text {. }
$$

Similarly, if $\cos (2 \pi b / a)$ is positive, then the second term of (11) is no more than $3 \cos (2 \pi b / a) / \sqrt{k} e^{4 \pi k}$, and if $\cos (2 \pi b / a)$ is negative, the bound is

$$
\frac{3 \cos (2 \pi b / a)}{\sqrt{k} e^{4 \pi k}}\left[1-\frac{1}{32 \pi k}\right] .
$$

Plugging these into (11), we get the bounds that are used in the secondary program; this gave us a positive lower bound on $U(s)$ for all but nine of the moduli, the outliers being 119299 192, 124414899 , $157619067,172672063,174523771,175990483$, 177351128,192214136 , and 246616980.

Equation (12) is the tip of the iceberg; in fact, we have a whole family of inequalities of this type. The relevant one for us comes from [1] and approximates $K_{v}(2 \pi k n)$ more sharply in some cases:

$$
\begin{aligned}
1-\frac{1-4 v^{2}}{16 \pi n k} & +\frac{\left(1-4 v^{2}\right)\left(9-4 v^{2}\right)}{2 !(16 \pi n k)^{2}}-\frac{\left(1-4 v^{2}\right)\left(9-4 v^{2}\right)\left(25-4 v^{2}\right)}{3 !(16 \pi n k)^{3}} \\
& \leq 2(k n)^{1 / 2} e^{2 \pi k n} K_{v}(2 \pi k n) \leq 1-\frac{1-4 v^{2}}{16 \pi n k}+\frac{\left(1-4 v^{2}\right)\left(9-4 v^{2}\right)}{2 !(16 \pi n k)^{2}} .
\end{aligned}
$$

For the nine remaining moduli, we divided up the $v$-range into ten equal parts and used the better of the above bounds (12) and (13) to bound the Bessel functions in each of these ranges. We also note the bounds

$$
n^{s-1 / 2} \sigma_{1-2 s}(n) \leq n^{1 / 2} \sigma_{-1}(n) \quad \text { and } \quad \sigma_{-1}(n) \leq \frac{3}{2}(n-1) \quad \text { for } n \geq 2,
$$

both of which are proven in [1]. These are used in conjunction with the above. For each $v$-range, $H(s)$ is estimated using these. In theory, if the lower bound were not positive, the $v$-range could be further divided or expansions with more terms could be used in place of (13), but for the nine moduli we considered at this stage, the above was sufficient to show condition (ii). 


\section{RESULTS}

The programs were run during off-peak hours on a SPARC over a 3-month period in 1997. As indicated above, we were able to show that (ii) always holds for the $d$ in our range, while failures of (i) and (iii) could be handled. In all cases, the programs were able to show that $L(s, \chi)$ had no positive real zeros, in accordance with the well-believed conjectures.

\section{REFERENCES}

[1] P. Bateman and E. Grosswald, On Epstein's zeta function. Acta Arith. 9 (1964), 365-373. MR 31:3392

[2] J. B. Conrey and K. Soundararajan, Real zeros of quadratic Dirichlet L-functions. Invent. Math. 150 (2002), 1-44.

[3] M. Low, Real zeros of the Dedekind zeta function of an imaginary quadratic field. Acta Arith. 14 (1968), 117-140. MR 38:4425

[4] G. Purdy, The real zeros of the Epstein zeta function. Ph. D. thesis. Univ. of Illinois (1972).

[5] G. Rieger, Review of [3, Math. Reviews 38/4425 (1970).

Department of Mathematics, McAllister Building, The Pennsylvania State UniverSity, University Park, Pennsylvania 16802

E-mail address: watkins@math.psu.edu 